\title{
Ring chromosome 6 syndrome
}

INSERM

\section{Source}

INSERM. (1999). Orphanet: an online rare disease and orphan drug data base. Ring chromosome 6 syndrome. ORPHA:1448

Ring chromosome 6 syndrome is a rare chromosomal anomaly syndrome with highly variable phenotype principally characterized by prenatal/postnatal growth failure, intellectual disability, developmental delay, craniofacial dysmorphism (incl. microcephaly, microphthalmia, epicanthus, low-set and malformed ears, broad and flat nasal bridge, full lips, micrognathia), central nervous system anomalies (e.g. hydrocephalus, cortical atrophy, ventriculomeg aly), short neck, and delayed bone age. Cardiac defects, limb anomalies, hip joint malformations, and seizures have also been reported. 